\title{
Heavy-Meson Decay Constants: Isospin Breaking from QCD Sum Rules
}

\author{
Wolfgang Lucha ${ }^{1, a}$, Dmitri Melikhov ${ }^{1,2,3, b}$, and Silvano Simula ${ }^{4, c}$ \\ ${ }^{1}$ Institute for High Energy Physics, Austrian Academy of Sciences, Nikolsdorfergasse 18, A-1050 Vienna, \\ Austria \\ ${ }^{2}$ D. V. Skobeltsyn Institute of Nuclear Physics, M. V. Lomonosov Moscow State University, 119991, Moscow, \\ Russia \\ ${ }^{3}$ Faculty of Physics, University of Vienna, Boltzmanngasse 5, A-1090 Vienna, Austria \\ ${ }^{4}$ INFN, Sezione di Roma Tre, Via della Vasca Navale 84, I-00146 Roma, Italy
}

\begin{abstract}
Compared with lattice QCD, isospin breaking of heavy-meson decay constants from QCD sum rules agrees for $D$ mesons but disagrees (by a factor of four) for $B$ mesons.
\end{abstract}

\section{Decay constants of heavy-light mesons from QCD sum-rule perspective}

QCD sum rules [1] constitute analytic relationships between the experimentally measurable properties of hadrons, on the one hand, and the fundamental parameters of the underlying quantum field theory of the strong interactions, quantum chromodynamics (QCD), on the other hand. They therefore represent a standard (occasionally perhaps indispensable) theoretical tool of hadron physics. Some time ago, we embarked on thorough investigations of both the accuracy and precision actually achievable by and the systematic uncertainties inherent to the formalism of QCD sum rules [2-6]. As a consequence of these studies, we proposed a somewhat modified algorithm that provides estimates of intrinsic errors [7-11]. With this improvement at our disposal, we revisited QCD sum-rule extractions of a variety of hadronic observables, particularly of the leptonic decay constants of the heavy-light mesons [12-16], which led us to the conclusion [17-21] that, in the bottom-meson system, the decay constants of the pseudoscalar mesons exceed those of their vector counterparts, as has been confirmed thereafter in lattice QCD [22].

For pseudoscalar $\left(P_{q}\right)$ and vector $\left(V_{q}\right)$ mesons $H_{q} \equiv P_{q}, V_{q}$, of mass $M_{H_{q}}$, built up by a heavy quark $Q=c, b$ and a light quark $q=u, d$ of masses $m_{Q}$ and $m_{q}$, respectively, the decay constants, $f_{H_{q}}$, of these mesons [with momentum $p$ and, in the case of vector mesons, polarization vector $\varepsilon_{\mu}(p)$ ] are defined, in terms of suitable, interpolating heavy-light axial-vector (A) and vector (V) quark-current operators, by

$$
\left\langle 0\left|\bar{q} \gamma_{\mu} \gamma_{5} Q\right| P_{q}(p)\right\rangle=\mathrm{i} f_{P_{q}} p_{\mu}, \quad\left\langle 0\left|\bar{q} \gamma_{\mu} Q\right| V_{q}(p)\right\rangle=f_{V_{q}} M_{V_{q}} \varepsilon_{\mu}(p) .
$$

Our actual aim [23, 24] is the impact of the isospin-spoiling disparity of the $u$ - and $d$-quark masses [25]

$$
m_{u}(2 \mathrm{GeV})=\left(2.3_{-0.5}^{+0.7}\right) \mathrm{MeV}, \quad m_{d}(2 \mathrm{GeV})=\left(4.8_{-0.3}^{+0.5}\right) \mathrm{MeV}
$$

on the differences $f_{H_{d}}-f_{H_{u}}$ of the decay constants of the charmed and beauty mesons $H=D, D^{*}, B, B^{*}$.

\footnotetext{
ae-mail: Wolfgang.Lucha@oeaw.ac.at

be-mail: dmitri_melikhov@gmx.de

e-mail: simula@roma3.infn.it
} 


\section{Dependence of heavy-light meson decay constants on light-quark mass}

Ignoring electromagnetic and weak interactions, we focus on the strong interactions of the light quarks $u$ and $d$, which then are basically characterized by just their masses $m_{u}$ and $m_{d}$, respectively. In order to track the dependence of the heavy-light meson decay constants on the light-quark masses, we consider a fictitious light quark $q$ of mass $m_{q}$ which we allow to vary from the average of the $u$ and $d$ masses [25]

$$
m_{u d} \equiv \frac{m_{u}+m_{d}}{2}, \quad m_{u d}=\left(3.5_{-0.2}^{+0.7}\right) \mathrm{MeV},
$$

up to the mass $m_{s}$ of the strange quark, $m_{s}=(95 \pm 5) \mathrm{MeV}$ [25]. Such generalization, however, enables us to introduce generic decay-constant functions $f_{H}\left(m_{q}\right)$ of that continuously varying light-quark mass $m_{q}$, derived from our QCD sum-rule approach such that the decay constants at the $m_{q}$ values of interest may be defined by the identification $f_{H_{u|d| l d}} \equiv f_{H}\left(m_{u|d| u d}\right)$. In terms of the $u$ - and $d$-quark mass difference $\delta m \equiv m_{d}-m_{u}>0$, the decay-constant differences $f_{H_{d}}-f_{H_{u}}$ thus emerge, at lowest order in $\delta m$, from the derivative of $f_{H}\left(m_{q}\right)$ with respect to $m_{q}$ in some vicinity of the $u$ and $d$ masses, say, at their average $m_{u d}$ :

$$
f_{H_{d}}-f_{H_{u}}=\left.\frac{\partial f_{H}\left(m_{q}\right)}{\partial m_{q}}\right|_{m_{q}=m_{u d}} \delta m+O\left(\delta m^{2}\right) .
$$

For simplicity, we prefer to introduce the shifted and rescaled, dimensionless light-quark mass variable

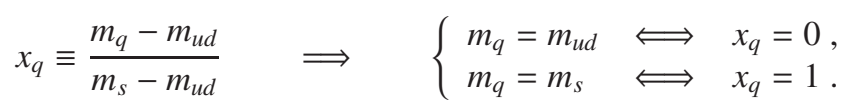

By benefitting from mutual cancellations of individual errors, a substantial diminution of uncertainties ought to be gained by discussing, instead of our decay-constant functions $f_{H}\left(x_{q}\right)$ themselves, the ratios

$$
R_{H}\left(x_{q}\right) \equiv \frac{f_{H}\left(x_{q}\right)}{f_{H_{u d}}}
$$

of the redefined decay-constant functions $f_{H}\left(x_{q}\right)$ and their values $f_{H}(0) \equiv f_{H_{u d}}$ at the mass average $m_{u d}$. The derivative of any such decay-constant ratio with respect to $x_{q}$, that is, its slope, at the origin $x_{q}=0$,

$$
\left.R_{H}^{\prime}(0) \equiv \frac{\mathrm{d} R_{H}\left(x_{q}\right)}{\mathrm{d} x_{q}}\right|_{x_{q}=0},
$$

then determines the $(\bar{Q} q)$ meson's decay-constant difference $f_{H_{d}}-f_{H_{u}}$, normalized to $f_{H_{u d}}$, according to

$$
\frac{f_{H_{d}}-f_{H_{u}}}{f_{H_{u d}}}=R_{H}^{\prime}(0) \frac{m_{d}-m_{u}}{m_{s}-m_{u d}} .
$$

\section{Heavy-light meson decay constants: improved QCD sum-rule formalism}

QCD sum rules arise from evaluation of vacuum expectation values of nonlocal products of convenient interpolating currents simultaneously at hadronic and QCD levels, by use of Wilson's operator product expansion for conversion of nonlocal operators into series of local ones, of Borel transformations from relevant momenta to Borel variables, generically called $\tau$, and of the quark-hadron duality assumption, asserting equality of hadron-state and perturbative-QCD contributions beyond effective thresholds $s_{\text {eff }}^{H_{q}}$. Taking into account the effective thresholds' Borel variable dependence $s_{\text {eff }}^{H_{q}}=s_{\text {eff }}^{H_{q}}(\tau)$ proves crucial for the accuracy of QCD sum-rule outcomes and our ability to estimate their intrinsic uncertainties [2-11]. Application of all these procedures to interpolating currents of type $J=\mathrm{A}, \mathrm{V}$ results in QCD sum rules 


$$
f_{H_{q}}^{2} M_{H_{q}}^{2} \exp \left(-M_{H_{q}}^{2} \tau\right)=\int_{\left(m_{Q}+m_{q}\right)^{2}}^{s_{\text {eff }}^{q}(\tau)} \mathrm{d} s \exp (-s \tau) \rho_{J}\left(s, m_{Q}, m_{q}, m_{\mathrm{sea}}, \alpha_{\mathrm{s}}\right)+\widehat{\Pi}_{J}\left(\tau, m_{Q}, m_{q},\langle\bar{q} q\rangle, \ldots\right)
$$

receiving contributions of purely perturbative origin, which may be represented by dispersion integrals of spectral densities $\rho_{J}\left(s, m_{Q}, m_{q}, m_{\text {sea }}, \alpha_{\mathrm{s}}\right)$, and nonperturbative contributions $\widehat{\Pi}_{J}\left(\tau, m_{Q}, m_{q},\langle\bar{q} q\rangle, \ldots\right)$ parametrized by so-called vacuum condensates (vacuum expectation values of colour-singlet operators constructed from the degrees of freedom of QCD) that characterize the properties of the QCD vacuum.

Picking up the terminology of lattice-QCD practitioners, in the operator product expansion we take the liberty to discriminate notationally those quarks which compose the interpolating currents from the "sea" quarks that contribute only to radiative corrections and denote all the masses of the latter by $m_{\text {sea. }}$. As perturbative series expansions in powers of the strong coupling $\alpha_{\mathrm{s}}$, our spectral densities are known [26-29] fully up to two-loop order $O\left(\alpha_{\mathrm{s}}\right)$ but at three-loop order $O\left(\alpha_{\mathrm{s}}^{2}\right)$ only for the case $m_{q}=m_{\text {sea }}=0$.

Following Ref. [28], we ensure perturbative convergence of our QCD sum-rule results by adopting for the definition of the quark masses the modified minimal-subtraction $(\overline{\mathrm{MS}})$ renormalization scheme.

Physical quantities, such as decay constants, cannot depend on unphysical (renormalization) scales introduced on calculational grounds. Nevertheless, procedures required by the application of the QCD sum-rule formalism induce for several reasons artificial scale dependences of the resulting predictions:

- Unavoidable truncations of perturbative expansions produce scale dependences of spectral densities.

- Effective thresholds are determined at given scales such as to reproduce experimental meson masses.

Although implementation of our advanced QCD sum-rule algorithms to heavy-meson decay constants removes a large portion of such scale dependences [12-21], remaining scale dependences contribute to the systematic uncertainties of any extracted QCD sum-rule outcomes. Consequently, in the following we present our findings for the scales $\mu$ at which the quoted estimates have been obtained. These scales are $\mu=1.7 \mathrm{GeV}$ for the charmed mesons $D$ and $D^{*}$ and $\mu=3.75 \mathrm{GeV}$ for the bottom mesons $B$ and $B^{*}$.

The numerical values of the set of parameters employed as input to our operator product expansion are given in Table 1. In addition, we need an idea about how the mass $M_{H_{q}}$ of the fictitious $(\bar{Q} q)$ meson $H_{q}$ and the vacuum condensate $\langle\bar{q} q\rangle$ of that fictitious light quark $q$ behave with varying quark mass $m_{q}$ :

Table 1. Parameter values relevant for our QCD sum-rule analysis of the decay constants of heavy-light mesons.

\begin{tabular}{lcr}
\hline Parameter in operator product expansion & Numerical input value & References \\
\hline$m_{u d}(2 \mathrm{GeV})$ & $(3.70 \pm 0.17) \mathrm{MeV}$ & {$[30,31]$} \\
$m_{s}(2 \mathrm{GeV})$ & $(93.9 \pm 1.1) \mathrm{MeV}$ & {$[30,31]$} \\
$m_{c}\left(m_{c}\right)$ & $(1275 \pm 25) \mathrm{MeV}$ & {$[25]$} \\
$m_{b}\left(m_{b}\right)$ & $(4247 \pm 34) \mathrm{MeV}$ & {$[14]$} \\
$\alpha_{\mathrm{s}}\left(M_{Z}\right)$ & $0.1184 \pm 0.0020$ & {$[13,15,20]$} \\
$\langle\bar{\ell} \ell\rangle(2 \mathrm{GeV}) \equiv \frac{\langle\bar{u} u\rangle+\langle\bar{d} d\rangle}{2}$ & $-\left[(267 \pm 17) \mathrm{MeV}^{3}\right.$ & {$[13,15,20,28]$} \\
$\frac{\langle\bar{s} s\rangle(2 \mathrm{GeV})}{\langle\bar{\ell} \ell\rangle(2 \mathrm{GeV})}$ & $0.8 \pm 0.3$ & {$[13,15,20,28]$} \\
$\left\langle\frac{\alpha_{\mathrm{s}}}{\pi} G G\right\rangle$ & $(0.024 \pm 0.012) \mathrm{GeV}^{4}$ & {$[13,15,20,28]$} \\
$\frac{\left\langle\bar{\ell} g_{\mathrm{s}} \sigma G \ell\right\rangle(2 \mathrm{GeV})}{\langle\bar{\ell} \ell\rangle(2 \mathrm{GeV})}$ & $(0.8 \pm 0.2) \mathrm{GeV}^{2}$ & {$[13,15,20,28]$} \\
\hline
\end{tabular}


- Lattice QCD [32-35] hints at a linear rise of the masses of charmed and bottom mesons with $m_{q}$. So, for the masses $M_{H_{q}}\left(x_{q}\right)$ of the heavy-light $(\bar{Q} q)$ mesons, we assume a linear $x_{q}$ dependence from the measured [25] nonstrange-meson mass $M_{H_{u d}}$ up to the corresponding strange-meson mass $M_{H_{s}}$ [25]:

$$
M_{H_{q}}\left(x_{q}\right)=M_{H_{u d}}+x_{q}\left(M_{H_{s}}-M_{H_{u d}}\right) .
$$

- For the light-quark condensate $\langle\bar{q} q\rangle$, we assume a linear $x_{q}$ dependence from $\langle\bar{\ell} \ell\rangle \equiv(\langle\bar{u} u\rangle+\langle\bar{d} d\rangle) / 2$ at $m_{u d} \equiv\left(m_{u}+m_{d}\right) / 2$ [cf. Eq. (1)] up to the strange-quark condensate $\langle\bar{s} s\rangle$ at $m_{s}=(95 \pm 5) \mathrm{MeV}$ [25]:

$$
\langle\bar{q} q\rangle=\langle\bar{\ell} \ell\rangle+x_{q}(\langle\bar{s} s\rangle-\langle\bar{\ell} \ell\rangle) .
$$

For the determination of the dependence of the effective thresholds $s_{\text {eff }}^{H_{q}}(\tau)$ on the Borel variable $\tau$, it proves to be sufficient to model their behaviour by polynomial ansätze with expansion coefficients $s_{j}^{(n)}$,

$$
s_{\mathrm{eff}}^{H_{q}}(\tau)=\sum_{j=0}^{n} s_{j}^{(n)} \tau^{j},
$$

of rather low order $n=1,2,3$, optimized by requiring a satisfactory reproduction of the experimentally measured meson masses $M_{H_{q}}$ by the corresponding QCD sum-rule predictions [7-11]. At a given mass

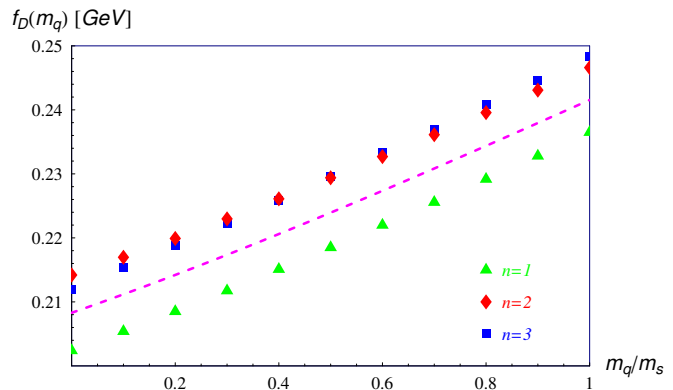

(a)

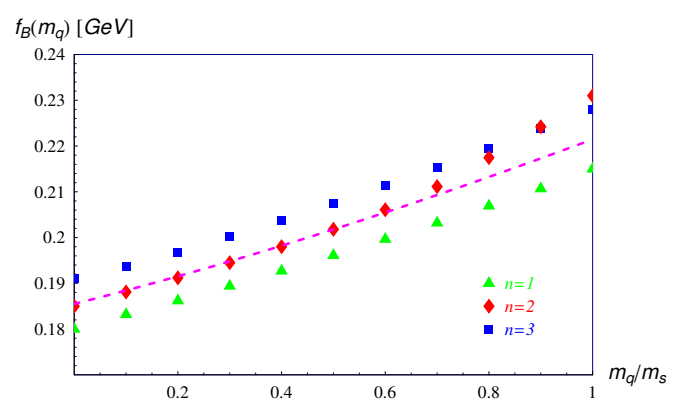

(c)

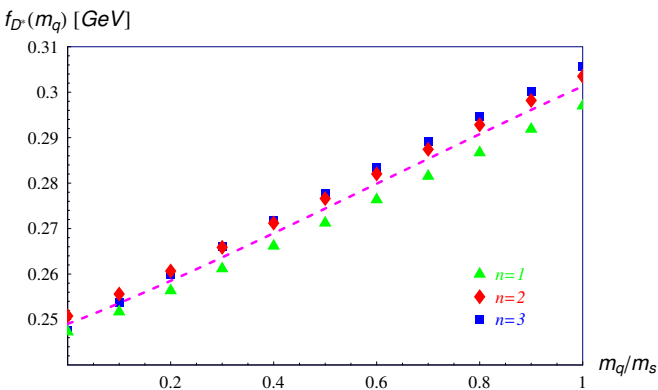

(b)

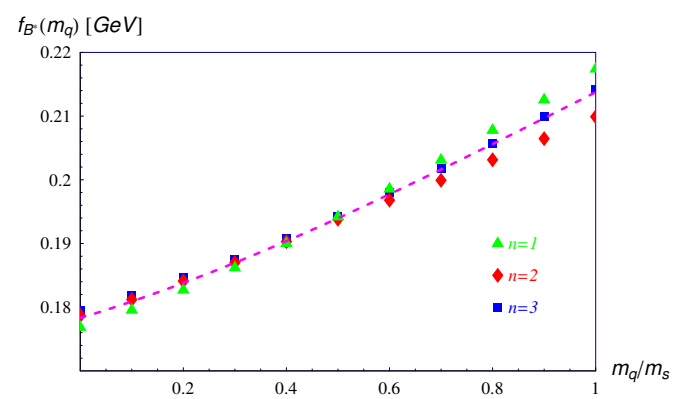

(d)

Figure 1. Dependence on the light-quark mass $m_{q}$ normalized to the strange-quark mass $m_{s}$ of the decay constants $f_{H}\left(m_{q}\right)$ of heavy-light $(\bar{Q} q)$ mesons of (a) $D$, (b) $D^{*}$, (c) $B$ and (d) $B^{*}$ type predicted [23], with centers indicated by dashed magenta lines, by QCD sum rules [7-11] relying on polynomial ansätze of order $n=1$ (green triangles $\triangle$ ), $n=2$ (red diamonds $\downarrow$ ), and $n=3$ (blue squares $\square$ ) for the Borel-parameter-dependent effective thresholds $s_{\text {eff }}^{H_{q}}(\tau)$. 


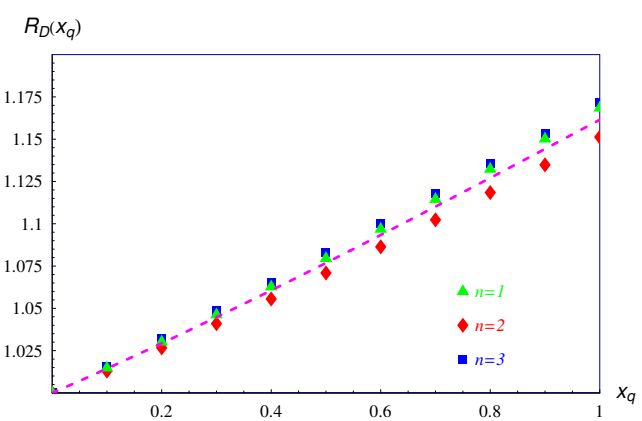

(a)

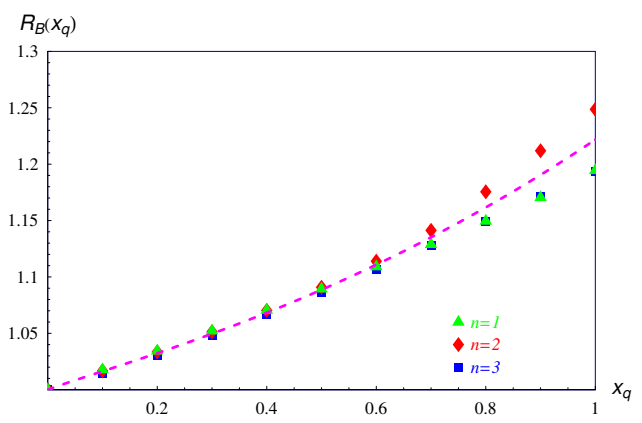

(c)

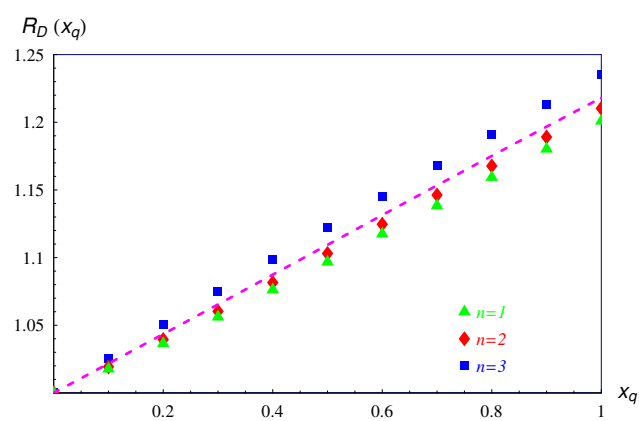

(b)

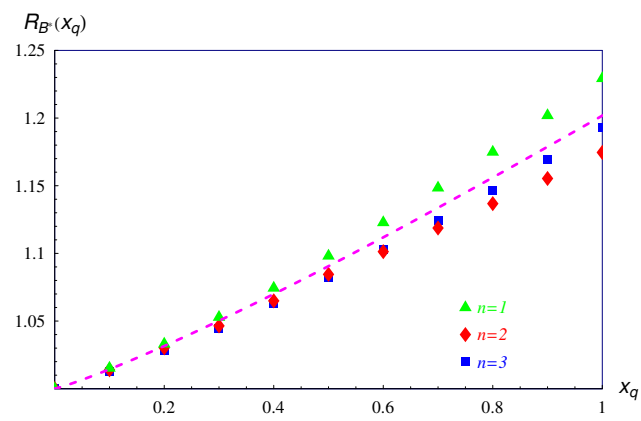

(d)

Figure 2. Dependence on the light-quark mass ratio $x_{q}$ in Eq. (2) of the ratios $R_{H}\left(x_{q}\right) \equiv f_{H}\left(x_{q}\right) / f_{H}(0)$ of the decay constants for heavy-light $(\bar{Q} q)$ mesons of (a) $D$, (b) $D^{*}$, (c) $B$ and (d) $B^{*}$ type found [24], with centers indicated by dashed magenta lines, by QCD sum rules [7-11] relying on polynomial ansätze of order $n=1$ (green triangles $\triangle$ ), $n=2$ (red diamonds $\downarrow$ ), and $n=3$ (blue squares $\square$ ) for the Borel-parameter-dependent effective thresholds $s_{\text {eff }}^{H_{q}}(\tau)$.

value $m_{q}$, the spread of predictions, obtained by application of our QCD sum-rule algorithms along the lines detailed in our earlier decay-constant extractions [12-16], for the case of linear $(n=1)$, quadratic $(n=2)$, and cubic $(n=3)$ behaviour of the effective thresholds $s_{\text {eff }}^{H_{q}}(\tau)$ provides, by its central value and half-width, estimates of the decay-constant functions $f_{H}\left(m_{q}\right)$ and ratios $R_{H}\left(x_{q}\right)$, Eq. (3), as well as their systematic uncertainties [7-11], shown for the mesons $H_{q}=D, D^{*}, B, B^{*}$ in Figs. 1 and 2, respectively.

\section{Decay-constant differences from the slopes of decay-constant functions}

In order to eventually extract from the QCD sum-rule outcomes in Fig. 2 the slopes $R_{H}^{\prime}(0)$ determining, as expressed by Eq. (4), the decay-constant differences $f_{H_{d}}-f_{H_{u}}$, we describe the behaviour of any ratio $R_{H}\left(x_{q}\right)$ as function of the mass-ratio variable $x_{q}$ in its respective $x_{q}$ interval, in three different ways, two of naïve polynomial shape and one inspired by heavy-meson chiral perturbation theory (HM $\chi \mathrm{PT})[36]$ :

$$
\begin{array}{llrl}
R_{H}\left(x_{q}\right)=1+a^{H} x_{q}, & 0<x_{q}<0.4 & \text { (linear ansatz) }, \\
R_{H}\left(x_{q}\right)=1+b_{1}^{H} x_{q}+b_{2}^{H} x_{q}^{2}, & 0<x_{q}<1 & \text { (quadratic ansatz) }, \\
R_{H}\left(x_{q}\right)=1+R_{\chi}\left(m_{q}, m_{u d}, m_{s}\right)+c_{1}^{H} x_{q}+c_{2}^{H} x_{q}^{2}, & 0<x_{q}<1 & \text { (HM } \chi \text { PT ansatz) } .
\end{array}
$$


In the $\mathrm{HM} \chi \mathrm{PT}$ case, the term $R_{\chi}\left(m_{q}, m_{u d}, m_{s}\right)$ is independent of the mesons $H_{q}$ under consideration; its (somewhat lengthy) explicit expression can be found in the Appendix of Ref. [24]. The parameters $a^{H}$, $b_{i}^{H}$ and $c_{i}^{H}(i=1,2)$ may be utilized to optimize our fits. Table 2 summarizes our findings for the slopes $R_{H}^{\prime}(0)$, derived by fitting the $x_{q}$ dependences of the ratios $R_{H}\left(x_{q}\right)$ depicted in Fig. 2 for each of the three ansätze (5a), (5b), and (5c), as well as the averages of the three individual results, for $H=D, D^{*}, B, B^{*}$.

Table 2. Numerical values of the decay-constant-governing slope $R_{H}^{\prime}(0)$ of the light-quark-mass dependent ratio $R_{H}\left(x_{q}\right)$ at the average $u-d$ mass point $x_{q}=0$, resulting from the three fits given by Eqs. (5a), (5b) and (5c) in their respective $x_{q}$ intervals, and of the corresponding averages for the charmed and bottom mesons $H=D, D^{*}, B, B^{*}$.

\begin{tabular}{lcccc}
\hline Meson & & \multicolumn{2}{c}{$R_{H}^{\prime}(0)$} \\
& linear fit (5a) & quadratic fit $(5 \mathrm{~b})$ & $\mathrm{HM} \chi \mathrm{PT}$ fit $(5 \mathrm{c})$ & average \\
& $0<x_{q}<0.4$ & $0<x_{q}<1$ & $0<x_{q}<1$ & \\
\hline$D$ & $0.148 \pm 0.007$ & $0.144 \pm 0.009$ & $0.171 \pm 0.009$ & $0.154 \pm 0.015$ \\
$D^{*}$ & $0.218 \pm 0.016$ & $0.218 \pm 0.021$ & $0.248 \pm 0.022$ & $0.228 \pm 0.024$ \\
$B$ & $0.168 \pm 0.004$ & $0.146 \pm 0.008$ & $0.174 \pm 0.008$ & $0.163 \pm 0.014$ \\
$B^{*}$ & $0.156 \pm 0.006$ & $0.139 \pm 0.009$ & $0.162 \pm 0.010$ & $0.152 \pm 0.013$ \\
\hline
\end{tabular}

For the lattice-QCD value $\delta m(2 \mathrm{GeV})=(2.67 \pm 0.22) \mathrm{MeV}[30,31]$ of the $u$ - $d$ mass difference and the averages of Table 2, Eq. (4) predicts, for the normalized decay-constant differences $\left(f_{H_{d}}-f_{H_{u}}\right) / f_{H_{u d}}$,

$$
\begin{aligned}
\frac{f_{D^{ \pm}}-f_{D^{0}}}{f_{D}}=0.0046(6), & \frac{f_{D^{* \pm}}-f_{D^{* 0}}}{f_{D^{*}}}=0.0067(9), \\
\frac{f_{B^{0}}-f_{B^{ \pm}}}{f_{B}}=0.0048(6), & \frac{f_{B^{* 0}}-f_{B^{* \pm}}}{f_{B^{*}}}=0.0045(5),
\end{aligned}
$$

indicating that the decay constants satisfy the inequality $f_{H_{d}}>f_{H_{u}}$ for all the mesons $H=D, D^{*}, B, B^{*}$. Multiplication of the above ratios by the decay constants collected in Table 3 entails, as our final results for the decay-constant differences of charmed and bottom heavy-light mesons due to isospin breaking,

$$
\begin{aligned}
f_{D^{ \pm}}-f_{D^{0}}=(0.95 \pm 0.13) \mathrm{MeV}, & f_{D^{* \pm}}-f_{D^{* 0}}=(1.69 \pm 0.27) \mathrm{MeV} \\
f_{B^{0}}-f_{B^{ \pm}}=(0.92 \pm 0.13) \mathrm{MeV}, & f_{B^{* 0}}-f_{B^{* \pm}}=(0.82 \pm 0.11) \mathrm{MeV}
\end{aligned}
$$

Table 3. Decay constants $f_{H}$ of the charmed and beauty mesons $H=D, D^{*}, B, B^{*}$ : numerical values [13-15, 20].

\begin{tabular}{lcr}
\hline Meson $H$ & Decay constant $f_{H}(\mathrm{MeV})$ & Reference \\
\hline$D$ & $206.2 \pm 8.9$ & {$[13]$} \\
$D^{*}$ & $252.2 \pm 22.7$ & {$[15]$} \\
$B$ & $192.0 \pm 14.6$ & {$[14]$} \\
$B^{*}$ & $181.8 \pm 13.7$ & {$[20]$} \\
\hline
\end{tabular}

Table 4 confronts our predictions [24] with available lattice-QCD outcomes for the decay-constant differences $f_{H_{d}}-f_{H_{u}}$ of pseudoscalar heavy-light mesons: In the case of the $D$ mesons, we find a really nice agreement. In the case of the $B$ mesons, however, our QCD sum-rule predictions are by a factor of four smaller than respective lattice-QCD claims, betraying a tension of some three standard deviations. Confidence in our approach may be drawn from the fact that our results are of very similar size as those reported by lattice QCD for the $K$ mesons [41]: both systems lead to differences of the order of $1 \mathrm{MeV}$. 
Table 4. Decay-constant difference of pseudoscalar heavy mesons: QCD sum rule [24] vs. lattice QCD [37-40].

\begin{tabular}{lccr}
\hline Decay-constant difference & QCD sum rule [24] & Lattice QCD & References \\
\hline$f_{D^{ \pm}}-f_{D^{0}}(\mathrm{MeV})$ & $0.95 \pm 0.13$ & $0.94_{-0.12}^{+0.50}$ & {$[37]$} \\
$f_{B^{0}}-f_{B^{ \pm}}(\mathrm{MeV})$ & $0.92 \pm 0.13$ & $3.8 \pm 1.0$ & {$[38-40]$} \\
\hline
\end{tabular}

\section{Acknowledgements}

D. M. would like to express gratitude for support by the Austrian Science Fund (FWF) under project P29028-N27.

\section{References}

[1] M. A. Shifman, A. I. Vainshtein, and V. I. Zakharov, Nucl. Phys. B 147 (1979) 385.

[2] W. Lucha, D. Melikhov, and S. Simula, Phys. Rev. D 76 (2007) 036002, arXiv:0705.0470 [hep$\mathrm{ph}]$.

[3] W. Lucha, D. Melikhov, and S. Simula, Phys. Lett. B 657 (2007) 148, arXiv:0709.1584 [hep-ph].

[4] W. Lucha, D. Melikhov, and S. Simula, Phys. At. Nucl. 71 (2008) 1461.

[5] W. Lucha, D. Melikhov, and S. Simula, Phys. Lett. B 671 (2009) 445, arXiv:0810.1920 [hep-ph].

[6] D. Melikhov, Phys. Lett. B 671 (2009) 450, arXiv:0810.4497 [hep-ph].

[7] W. Lucha, D. Melikhov, and S. Simula, Phys. Rev. D 79 (2009) 096011, arXiv:0902.4202 [hep$\mathrm{ph}]$.

[8] W. Lucha, D. Melikhov, and S. Simula, J. Phys. G 37 (2010) 035003, arXiv:0905.0963 [hep-ph].

[9] W. Lucha, D. Melikhov, H. Sazdjian, and S. Simula, Phys. Rev. D 80 (2009) 114028, arXiv: 0910.3164 [hep-ph].

[10] W. Lucha, D. Melikhov, and S. Simula, Phys. Lett. B 687 (2010) 48, arXiv:0912.5017 [hep-ph].

[11] W. Lucha, D. Melikhov, and S. Simula, Phys. At. Nucl. 73 (2010) 1770, arXiv:1003.1463 [hep$\mathrm{ph}]$.

[12] W. Lucha, D. Melikhov, and S. Simula, J. Phys. G 38 (2011) 105002, arXiv:1008.2698 [hep-ph].

[13] W. Lucha, D. Melikhov, and S. Simula, Phys. Lett. B 701 (2011) 82, arXiv:1101.5986 [hep-ph].

[14] W. Lucha, D. Melikhov, and S. Simula, Phys. Rev. D 88 (2013) 056011, arXiv:1305.7099 [hep$\mathrm{ph}]$.

[15] W. Lucha, D. Melikhov, and S. Simula, Phys. Lett. B 735 (2014) 12, arXiv:1404.0293 [hep-ph].

[16] W. Lucha, D. Melikhov, and S. Simula, EPJ Web Conf. 80 (2014) 00043, arXiv:1407.5512 [hep$\mathrm{ph}]$.

[17] W. Lucha, D. Melikhov, and S. Simula, EPJ Web Conf. 80 (2014) 00046, arXiv:1410.6684 [hep$\mathrm{ph}$.

[18] W. Lucha, D. Melikhov, and S. Simula, arXiv:1411.3890 [hep-ph].

[19] W. Lucha, D. Melikhov, and S. Simula, AIP Conf. Proc. 1701 (2016) 050007, arXiv:1411.7844 [hep-ph].

[20] W. Lucha, D. Melikhov, and S. Simula, Phys. Rev. D 91 (2015) 116009, arXiv:1504.03017 [hep-ph].

[21] W. Lucha, D. Melikhov, and S. Simula, PoS (EPS-HEP2015) 532, arXiv:1508.07595 [hep-ph].

[22] B. Colquhoun et al., HPQCD Coll., Phys. Rev. D 91 (2015) 114509, arXiv:1503.05762 [hep-lat].

[23] W. Lucha, D. Melikhov, and S. Simula, HEPHY-PUB 971/16 (2016), arXiv:1609.02382 [hep$\mathrm{ph}]$. 
[24] W. Lucha, D. Melikhov, and S. Simula, HEPHY-PUB 973/16 (2016), RM3-TH/16-9, arXiv: 1609.05050 [hep-ph].

[25] K. A. Olive et al., Particle Data Group, Chin. Phys. C 38 (2014) 090001.

[26] K. G. Chetyrkin and M. Steinhauser, Phys. Lett. B 502 (2001) 104, arXiv:hep-ph/0012002.

[27] K. G. Chetyrkin and M. Steinhauser, Eur. Phys. J. C 21 (2001) 319, arXiv:hep-ph/0108017.

[28] M. Jamin and B. O. Lange, Phys. Rev. D 65 (2002) 056005, arXiv:hep-ph/0108135.

[29] P. Gelhausen, A. Khodjamirian, A. A. Pivovarov, and D. Rosenthal, Phys. Rev. D 88 (2013) 014015, arXiv:1305.5432 [hep-ph]; 89 (2014) 099901(E); 91 (2015) 099901(E).

[30] S. Aoki et al., FLAG Working Group, Eur. Phys. J. C 74 (2014) 2890, arXiv:1310.8555 [hep-lat].

[31] S. Aoki et al., FLAG Working Group, arXiv:1607.00299 [hep-lat].

[32] B. Blossier et al., Phys. Rev. D 82 (2010) 114513, arXiv:1010.3659 [hep-lat].

[33] P. Dimopoulos et al., ETM Coll., J. High Energy Phys. 01 (2012) 046, arXiv:1107.1441 [heplat].

[34] N. Carrasco et al., ETM Coll., Nucl. Phys. B 887 (2014) 19, arXiv:1403.4504 [hep-lat].

[35] A. Bussone et al., ETM Coll., Phys. Rev. D 93 (2016) 114505, arXiv:1603.04306 [hep-lat].

[36] S. R. Sharpe and Y. Zhang, Phys. Rev. D 53 (1996) 5125, arXiv:hep-lat/9510037.

[37] A. Bazavov et al., Fermilab Lattice and MILC Collaborations, Phys. Rev. D 90 (2014) 074509, arXiv:1407.3772 [hep-lat].

[38] R. J. Dowdall et al., HPQCD Coll., Phys. Rev. Lett. 110 (2013) 222003, arXiv:1302.2644 [heplat].

[39] N. H. Christ et al., RBC and UKQCD Collaborations, Phys. Rev. D 91 (2015) 054502, arXiv:1404.4670 [hep-lat].

[40] J. L. Rosner, S. Stone, and R. S. Van der Water, arXiv:1509.02220 [hep-ph].

[41] N. Carrasco et al., ETM Coll., Phys. Rev. D 91 (2015) 054507, arXiv:1411.7908 [hep-lat]. 